\title{
Mediastinal cyst as a cause of severe airway compression and dysphonia
}

\author{
Cisto mediastinal como causa de grave compressão da via aérea \\ central e disfonia
}

\begin{abstract}
Vanessa Costa Menezes, Paulo Francisco Guerreiro Cardoso, Hélio Minamoto, Márcia Jacomelli, Paulo Sampaio Gutierrez, Fabio Biscegli Jatene
\end{abstract}

\section{To the Editor:}

Extrinsic compression of the central airway is usually related to the presence of adjacent solid lesions, the most common being primary or secondary mediastinal tumors. Cystic lesions account for $12-30 \%$ of mediastinal masses and are usually benign and asymptomatic. Obstructive or compressive symptoms are extremely uncommon..$^{(1)}$

Here, we describe the case of a 56-year-old man with a 6-week history of progressive dyspnea who presented with dry cough and hoarseness. He had no fever, dysphagia, or weight loss. He was a former smoker (20 pack-years) and reported no history of surgical intervention or tracheal intubation. Physical examination revealed that the patient's blood pressure was $165 \times 95 \mathrm{mmHg}$, he had tachydyspnea at rest, dysphonia, and noisy respiration, and he was unable to tolerate the supine position; there was a hard, painless, smooth-surfaced swelling at the left supraclavicular fossa, the thyroid gland was not palpable, and there was no lymphadenopathy in the neck. Heart and lung auscultation was normal.

$A$ chest $X$-ray revealed mediastinal widening and tracheal deviation to the right. A chest CT scan revealed a cystic lesion without capsular calcification, measuring $12.1 \times 7.3 \times 7.2 \mathrm{~cm}$. The lesion was located in the middle mediastinum and caused compression of the mediastinal trachea, deviating it to the right and reducing its caliber. The unilocular cystic lesion extended to the superior mediastinal aperture, adjacently to the left lobe of the thyroid gland, displacing the esophagus to the left (Figure 1). The results of laboratory and thyroid function testing, as well as the marker levels, were within the normal range. Esophagogastroduodenoscopy, which was performed with the patient in a semi-sitting position because of severe dyspnea, revealed distal erosive esophagitis, severe erosive antral gastritis, and no compression or deviation of the esophagus.
The presumptive diagnosis of bronchogenic cyst or thyroid goiter with compression of the airway and associated vocal dysfunction was regarded as an indication for video-assisted flexible laryngotracheal bronchoscopy under topical anesthesia with intubation under bronchoscopic visualization, because of dyspnea, during the same anesthesia. The examination revealed paresis of the left vocal fold, which remained abducted; severe extrinsic tracheal compression with narrowing of the tracheal lumen in its proximal two-thirds; and architectural distortion of the distal trachea with normal mucosa. The patient then underwent orotracheal intubation guided by video-assisted bronchoscopy, was anesthetized, was placed in the left lateral decubitus position, and underwent right posterolateral thoracotomy in the fourth intercostal space with preservation of the latissimus dorsi muscle. A $30^{\circ}$ endoscope (Karl Storz Instruments, Tuttlingen, Germany) was inserted into the right seventh intercostal space, on the posterior axillary line, and revealed an encapsulated, cystic mediastinal lesion, measuring $12 \mathrm{~cm}$ in diameter, in the visceral compartment above the azygos vein, between the trachea and the esophagus. Resection consisted of a complete, video-assisted extracapsular dissection of the anterior, posterior, and inferior edges of the cyst, with preservation of the right recurrent laryngeal nerve.

Dyspnea resolved in the immediate postoperative period, and hoarseness resolved on postoperative day 2. The patient was discharged on postoperative day 4. Histopathology revealed a thin, elastic cyst wall composed of cuboidal cells arranged linearly among columnar epithelial cells. Immunohistochemical analysis was positive for parathyroid hormone, confirming the diagnosis of benign, nonfunctioning parathyroid cyst (Figure 2). 
Parathyroid cysts are generally asymptomatic and mimic thyroid lesions. Symptomatic parathyroid cysts are extremely rare, with about 200 cases having been reported. Of those, only 10 had recurrent laryngeal nerve paralysis and dysphonia. ${ }^{(1,2)}$ Parathyroid cysts are slow growing and are usually seen posteriorly to the left lobe of the thyroid gland, a common ground for intrathoracic thyroid goiters. Approximately $80 \%$ of these lesions are nonfunctioning and asymptomatic. ${ }^{(3)}$ Functioning cysts usually manifest as hyperparathyroidism caused by abnormal parathyroid hormone production leading to hypercalcemia. There has been one report of a large functioning parathyroid cyst mimicking a thyroid goiter with compressive symptoms. ${ }^{(4)}$ Compression of neighboring structures can lead to laryngeal stridor, dyspnea, and dysphagia; however, the combination of these factors in the presence of a nonfunctioning lesion, as in the case describe here, is extremely uncommon. The absence of dysphagia despite the large size of the cyst can be explained by the notorious capacity of the esophagus to accommodate itself in the mediastinum, even when compressed by large lesions.

The symptoms and characteristics of the patient's cyst are similar to those described by one group of authors, who performed
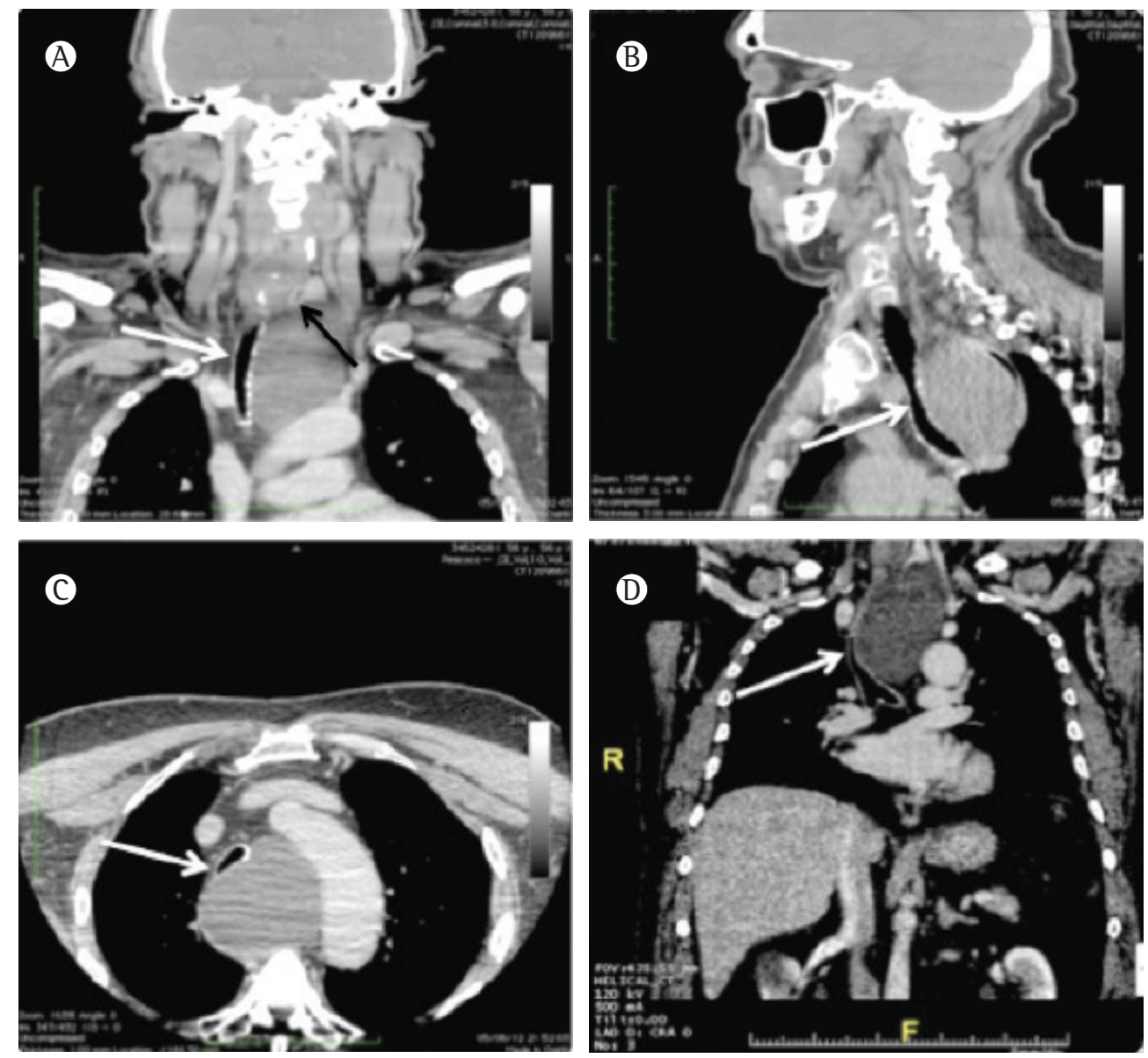

Figure 1 - Chest CT scans. In A, coronal reconstruction showing compression of the trachea (light arrow) by the lesion and contact of the lesion with the lower pole of the left lobe of the thyroid gland (dark arrow). In B, C, and D, respectively, sagittal, axial and coronal reconstructions showing a cystic lesion without calcification, located in the visceral compartment of the mediastinum and causing severe compression of the mediastinal trachea (light arrows). Note lack of intravenous contrast enhancement. 

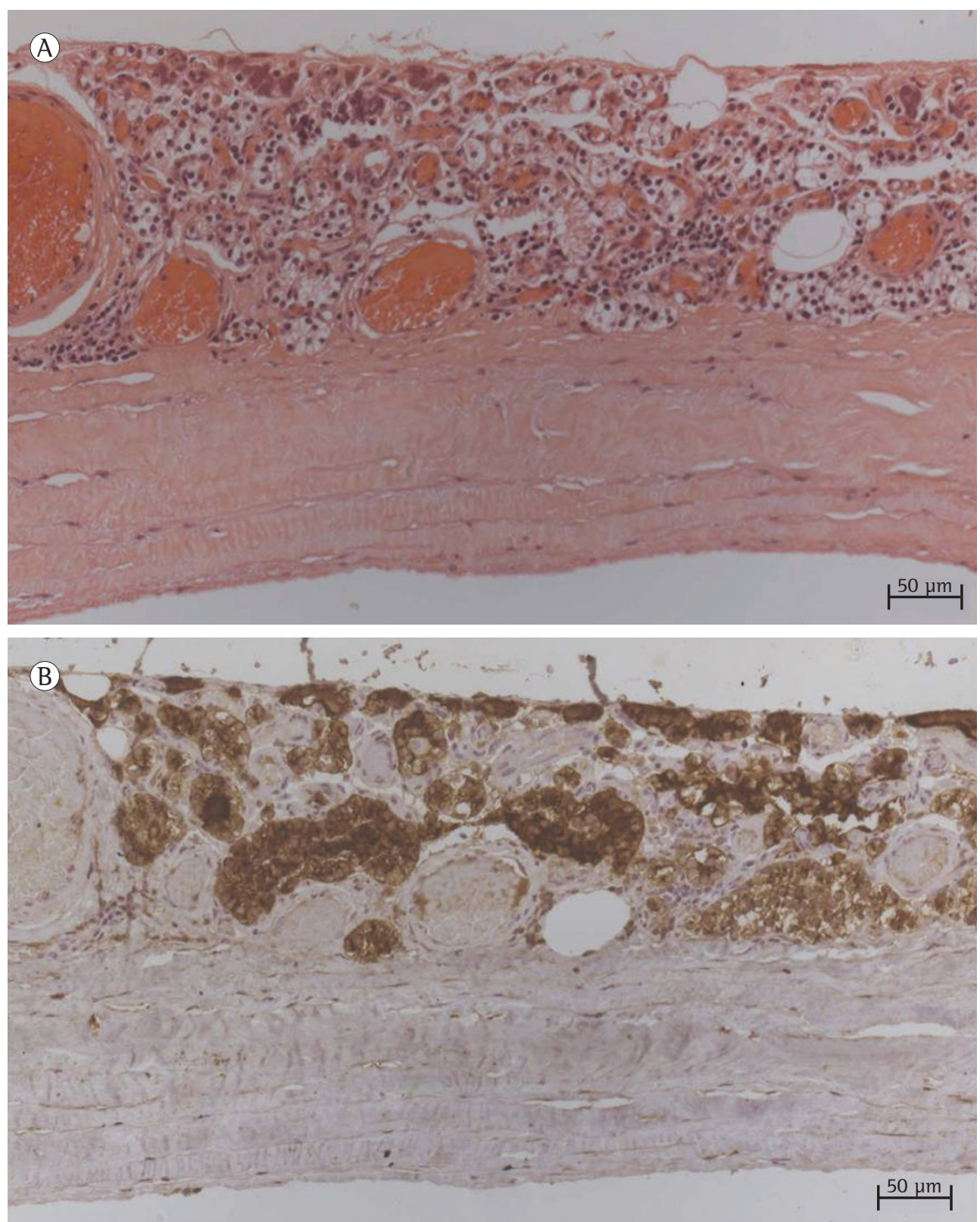

Figure 2 - In A, histological section of the cyst wall, which consisted of fibrous tissue covered by cuboidal epithelium (H\&E; magnification, $\times 20$ ). In B, positive immunohistochemical reaction for parathyroid hormone in the epithelial lining (magnification, $\times 20$ ).

a transcutaneous puncture to empty a cyst of its contents that resulted in subsequent recurrence, requiring surgical resection. ${ }^{(5)}$ Although there have been reports of cyst aspiration and sclerotherapy, this is not the recommended approach, which is why we did not perform it. In retrospect, however, it must be considered that, in the presence of severe airway obstruction, such as that described in our patient, the risk of this type of puncture would be reasonable in order to improve the ventilatory status until definitive treatment. Nevertheless, our choice to perform bronchoscopy and intubation under bronchoscopic visualization proved to be safe and effective. We did not perform scintigraphy or ultrasonography of the thyroid, because we considered that the results of these tests would not change the therapeutic approach. 
On CT scans, functioning cysts present as low-density homogeneous areas, whereas, on nuclear magnetic resonance imaging, they are seen as isointense areas because of their serous fluid content. In the case reported here, nuclear magnetic resonance imaging was not performed, because the $\mathrm{CT}$ findings were sufficient to establish the nature and limits of the cyst, especially in terms of its relationship with the mediastinal vascular structures.

There have been isolated reports of functioning cystic parathyroid carcinomas of the mediastinum. ${ }^{(6)}$ Cystic degeneration resulting from an adenoma or adenocarcinoma has a distinct behavior from that resulting from a parathyroid cyst. The content of an adenoma or adenocarcinoma is characterized predominantly by hemorrhagic or chocolate-like fluid, and the capsule is not surrounded by an epithelial layer. The content of a simple cyst is characterized by pearly clear fluid and is covered by a layer of flat columnar to cuboidal epithelial cells that have histological features typical of the parathyroid glands. ${ }^{(4)}$

Surgical resection is recommended for functioning cysts and for nonfunctioning cysts associated with compressive symptoms, in which case aspiration alone leads to recurrence of the lesion and symptoms. We chose to perform resection via video-assisted thoracotomy because of the topography of the visceral mediastinum and because of the need for detailed viewing of the structures so as to avoid injury to the right recurrent laryngeal nerve.

The present case demonstrates that, although nonfunctioning mediastinal parathyroid cysts are rare, they can be the cause of severe obstruction of the central airway and dysphonia, especially when they reach large sizes. Surgical treatment is the most effective and safe way to resolve compressive symptoms completely, and it should be preceded by careful diagnostic work-up and careful planning.

\section{Vanessa Costa Menezes}

Resident, Thoracic Surgery Section,

Heart Institute, University of São

Paulo School of Medicine

Hospital das Clínicas, São Paulo, Brazil
Paulo Francisco Guerreiro Cardoso Professor, Department of

Cardiorespiratory Diseases, Thoracic

Surgery Section, Heart Institute, University of São Paulo School of Medicine Hospital das Clínicas, São

Paulo, Brazil

\section{Hélio Minamoto}

Attending Physician, Thoracic Surgery Section, Heart Institute, University of

São Paulo School of Medicine Hospital das Clínicas, São Paulo, Brazil

Márcia Jacomelli

Attending Physician, Respiratory

Endoscopy Section, Heart Institute, University of São Paulo School of Medicine Hospital das Clínicas, São Paulo, Brazil

Paulo Sampaio Gutierrez Attending Physician, Anatomic Pathology Laboratory, Heart Institute, University of São Paulo School of Medicine Hospital das Clínicas, São Paulo, Brazil

\section{Fabio Biscegli Jatene}

Full Professor, Department of Cardiorespiratory Diseases, Thoracic Surgery Section, Heart Institute, University of São Paulo School of Medicine Hospital das Clínicas, São Paulo, Brazil

\section{References}

1. Landau O, Chamberlain DW, Kennedy RS, Pearson FG, Keshavjee S. Mediastinal parathyroid cysts. Ann Thorac Surg. 1997;63(4):951-3. http://dx.doi.org/10.1016/ S0003-4975(96)01392-6

2. Sánchez A, Carretto H. Treatment of a nonfunctioning parathyroid cyst with tetracycline injection. Head Neck. 1993;15(3):263-5. http://dx.doi.org/10.1002/hed.2880150317

3. Agrawal D, Lahiri TK, Agrawal A, Singh MK. Uncommon parathyroid mediastinal cyst compressing the trachea. Indian J Chest Dis Allied Sci. 2006;48(4):279-81. PMid:16970296

4. McKay GD, Ng TH, Morgan GJ, Chen RC. Giant functioning parathyroid cyst presenting as a retrosternal goitre. ANZ J Surg. 2007;77(4):297-304. http://dx.doi.org/10.1111/j.14452197.2007.04037.x PMid:17388841 
5. Umemori Y, Makihara S, Kotani K, Washio K. Mediastinal parathyroid cyst with tracheal constriction. Jpn J Thorac Cardiovasc Surg. 2002;50(2):85-7. http://dx.doi.org/10.1007/ BF02919671 PMid:11905065
6. Vazquez FJ, Aparicio LS, Gallo CG, Diehl M. Parathyroid carcinoma presenting as a giant mediastinal retrotracheal functioning cyst. Singapore Med J. 2007;48(11):e304-7. PMid:17975683

Submitted: 24 January 2013. Accepted, after review: 01 Febrary 2013. 